\title{
Commutators of the Fractional Hardy Operator on Weighted Variable Herz-Morrey Spaces
}

\author{
Amjad Hussain, ${ }^{1}$ Muhammad Asim, ${ }^{1}$ Muhammad Aslam, ${ }^{2}$ and Fahd Jarad $\left(\mathbb{D}{ }^{3,4}\right.$ \\ ${ }^{1}$ Department of Mathematics, Quaid-I-Azam University, Islamabad 44000, Pakistan \\ ${ }^{2}$ Department of Mathematics, College of Sciences, King Khalid University, Abha 61413, Saudi Arabia \\ ${ }^{3}$ Department of Mathematics, Çankaya University, 06790 Etimesgut, Ankara, Turkey \\ ${ }^{4}$ Department of Medical Research, China Medical University Hospital, China Medical University, Taichung, Taiwan
}

Correspondence should be addressed to Fahd Jarad; fahd@cankaya.edu.tr

Received 6 August 2021; Revised 14 September 2021; Accepted 16 September 2021; Published 19 October 2021

Academic Editor: Sarfraz Nawaz Malik

Copyright (c) 2021 Amjad Hussain et al. This is an open access article distributed under the Creative Commons Attribution License, which permits unrestricted use, distribution, and reproduction in any medium, provided the original work is properly cited.

In the present paper, our aim is to establish the boundedness of commutators of the fractional Hardy operator and its adjoint operator on weighted Herz-Morrey spaces with variable exponents $\operatorname{MK}_{p, q(\cdot)}^{\alpha(\cdot), \lambda}(w)$.

\section{Introduction}

Hardy operators and related commutators play an indispensable role in the theory of partial differential equations $[1,2]$ and the characterization of function spaces [3-5]. Without going into much details, let us first define the fractional Hardy operators [3]

$H g(z)=\frac{1}{|z|^{n-\beta}} \int_{|t| \leq|z|} g(t) d t, \quad H^{*} g(z)=\int_{|t|>|z|} \frac{g(t)}{|t|^{n-\beta}} d t, z \in \mathbb{R}^{n} /\{0\}$

and related commutators:

$$
\left[b, H_{\beta}\right] g=b H g-H(b g), \quad\left[b, H_{\beta}^{*}\right] g=b H^{*} g-H^{*}(b g) .
$$

It is important to note that taking $\beta=0$ in (1), we get multidimensional Hardy operator defined and studied in $[6,7]$. Also, (1) reduces to the one dimensional Hardy operator [8] if we choose $\beta=0$ and $n=1$. Here, we cite some important literature with regards to the study of Hardy-type operators on different function spaces which include [9-15].
The new development of variable exponent commenced with the work of Kov'a cik and R'akosn'ik in [16], where a class of function spaces having variable exponent was defined, and basic properties of variable exponent Lebesgue space were explored. Recently, the theory of variable exponent analysis is modeled in terms of the boundedness of the Hardy Littlewood maximal operator $M$ [17-21]:

$$
M g(z)=\sup _{B: \text { ball }, z \in B} \frac{1}{|B|} \int_{|B|} \int_{B}|g(t)| d t .
$$

Besides, Muckenhoupt $A_{p}$ theory [22] is generalized in the recent span of time with regard to variable exponent spaces ([23-28]). By taking into account the generalization of function spaces with variable exponents and the same with weights, many results like duality, boundedness of sublinear operators, the wavelet characterization, and commutators of fractional and singular integrals have been studied [29-38].

Recently, authors have studied generalized Herz space in terms of both Muckenhoupt weights and variable exponent [39-41]. Moreover, an idea of combining two function spaces to develop a new one is also an interesting problem in Harmonic analysis. One such problem is considered in [42] in which Herz-Morrey space was defined. Although, 
the weighted versions of Herz-Morrey spaces were introduced recently in $[43,44]$.

In this piece of work, our main focus is on establishing the boundedness of commutators of fractional Hardy operators on a class of function spaces called the weighted HerzMorrey space with variable exponents. We seek to find the boundedness of these commutators with symbol functions in BMO (bounded mean oscillation) spaces. In establishing such a boundedness, we make use of the boundedness of the fractional integral operator $I_{\beta}$

$$
I_{\beta}(g)(z)=\int_{\mathbb{R}^{n}} \frac{g(t)}{|z-t|^{n-\beta}} d t
$$

on weighted Lebesgue space which was done in [39].

In the rest of this paper, the symbol $C$ expresses a constant whose value may differ at all of its occurrences. The Greek letter $\chi_{S}$ denotes the characteristics function of a sphere $S$ where $S$ is a measurable subset of $R^{n}$ and $|S|$ represents its Lebesgue measure. Before turning to our key results, let us first define the relevant variable exponent function spaces.

\section{Preliminaries}

Let us consider a measurable function $p(\cdot)$ on $\mathbb{R}^{n}$ having range $[1, \infty)$. The Lebesgue space with variable exponent $L^{p(\cdot)}\left(\mathbb{R}^{n}\right)$ is the set of all measurable function $f$ such that

$$
L^{p(\cdot)}\left(\mathbb{R}^{n}\right)=\left\{f: \int_{\mathbb{R}^{n}}\left(\frac{|f(x)|}{\sigma}\right)^{p(x)} d x<\infty \text {, for some } \sigma>0\right\}
$$

The space $L^{p(\cdot)}\left(\mathbb{R}^{n}\right)$ turns out to be Banach function space under the norm:

$$
\|f\|_{L^{p(\cdot)}\left(\mathbb{R}^{n}\right)}=\inf \left\{\sigma>0: \int_{\mathbb{R}^{n}}\left(\frac{|f(x)|}{\sigma}\right)^{p(x)} d x \leq 1\right\} .
$$

We denote by $\mathscr{P}\left(\mathbb{R}^{n}\right)$ the set of all measurable functions $p(\cdot): \mathbb{R}^{n} \longrightarrow(1, \infty)$ such that

$$
1<p_{-} \leq p(x) \leq p_{+}<\infty
$$

where

$$
p_{-}:=\operatorname{essinf}_{x \in \mathbb{R}^{n}} p(x), p_{+}:=\operatorname{esssup}_{x \in \mathbb{R}^{n}} p(x)
$$

Definition 1. Suppose $p(\cdot)$ is a real valued function on $\mathbb{R}^{n}$. We say that

(i) $C_{l o c}^{\log }\left(\mathbb{R}^{n}\right)$ is the set of all local log-Holder continuous functions $p(\cdot)$ satisfying

$$
|p(x)-p(y)| \lesssim \frac{-C}{\log (|x-y|)}, \quad|x-y|<\frac{1}{2}, \quad x, y \in \mathbb{R}^{n} .
$$

(ii) $\mathscr{C}_{0}^{\log }\left(\mathbb{R}^{n}\right)$ is the set of all local log-Holder continuous function $p(\cdot)$ satisfying at the origin

$$
|p(x)-p(0)| \lesssim \frac{C}{\log (|e+(1 /|x|)|)}, \quad|x-y|<\frac{1}{2}, \quad x \in \mathbb{R}^{n} .
$$

(iii) $\mathscr{C}_{\infty}^{\log }\left(\mathbb{R}^{n}\right)$ is the set of all log-Holder continuous functions satisfying at infinity

$$
\left|p(x)-p_{\infty}\right| \leq \frac{C_{\infty}}{\log (e+|x|)}, \quad x \in \mathbb{R}^{n}
$$

(iv) $\mathscr{C}^{\log }\left(\mathbb{R}^{n}\right)=\mathscr{C}_{\infty}^{\log } \cap \mathscr{C}_{\text {loc }}^{\log }$ denotes the set of all global log-Holder continuous functions $p(\cdot)$.

It was proved in [21] that if $p(\cdot) \in \mathscr{P}\left(\mathbb{R}^{n}\right) \cap C^{\log }\left(\mathbb{R}^{n}\right)$, then Hardy-Littlewood maximal operator $M$ is bounded on $L^{p(\cdot)}\left(\mathbb{R}^{n}\right)$.

Suppose $w(x)$ is a weight function on $\mathbb{R}^{n}$, which is nonnegative and locally integrable on $\mathbb{R}^{n}$. Let $L^{p(\cdot)}(w)$ be the space of all complex-valued functions $f$ on $\mathbb{R}^{n}$ such that $f w^{1 / p(\cdot)} \in L^{p(\cdot)}\left(\mathbb{R}^{n}\right)$. The space $L^{p(\cdot)}(w)$ is a Banach function space equipped with the norm:

$$
\|f\|_{L^{p(\cdot)}(w)}=\left\|f w^{\frac{1}{p(\cdot)}}\right\|_{L^{p(\cdot)}}
$$

Benjamin Muckenhoupt introduced the theory of $A_{p}(1$ $<p<\infty)$ weights on $\mathbb{R}^{n}$ in [22]. Recently, in [39, 40], Izuki and Noi generalized the Muckenhoupt $A_{p}$ class by taking $p$ as a variable.

Definition 2. Let $p(\cdot) \in \mathscr{P}\left(\mathbb{R}^{n}\right)$. A weight $w$ is an $A_{p(\cdot)}$ weight if

$$
\sup _{B} \frac{1}{|B|}\left\|w^{1 / p(\cdot)} \chi_{B}\right\|_{L^{p(\cdot)}}\left\|w^{-1 / p(\cdot)} \chi_{B}\right\|_{L^{p^{\prime}(\cdot)}}<\infty
$$

In [25], the authors proved that $w \in A_{p(\cdot)}$ if and only if $M$ is bounded on the space $L^{p(\cdot)}$.

Remark 3 (see [39]). Suppose $p(\cdot), q(\cdot) \in \mathscr{P}\left(\mathbb{R}^{n}\right) \cap \mathscr{C}^{\log }\left(\mathbb{R}^{n}\right)$ and $p(\cdot) \leq q(\cdot)$, then we have

$$
A_{1} \subset A_{p(\cdot)} \subset A_{q(\cdot)} \cdot
$$

Definition 4. Suppose $p_{1}(\cdot), p_{2}(\cdot) \in \mathscr{P}\left(\mathbb{R}^{n}\right)$ and $\beta \in(0, n)$ such that $1 / p_{2}(x)=1 / p_{1}(x)-\beta / n$. A weight $w$ is said to be $A\left(p_{1}(\cdot), p_{2}(\cdot)\right)$ weight if

$$
\left\|\chi_{B}\right\|_{L^{p_{2}(\cdot)}\left(w^{p_{2}(\cdot)}\right)}\left\|\chi_{B}\right\|_{L^{p_{1}(\cdot)}\left(w^{p_{1}(\cdot)}\right)^{\prime}} \leq C|B|^{1-\frac{\beta}{n}}
$$


Definition 5 (see [39]). Suppose $p_{1}(\cdot), p_{2}(\cdot) \in \mathscr{P}\left(\mathbb{R}^{n}\right)$ and $\beta$ $\in(0, n)$ such that $1 / p_{2}(x)=1 / p_{1}(x)-\beta / n$. Then, $w \in$ $A_{\left(p_{1}(\cdot), p_{2}(\cdot)\right)}$ if and only if $w^{p_{2}(\cdot)} \in A_{1+p_{2}(\cdot) / p_{1}^{\prime}(\cdot)} \cdot$

Now, we define the variable exponent weighted MorreyHerz space $M \dot{K}_{q, p(\cdot)}^{\alpha(\cdot), \lambda}(w)$. Let $B_{k}=\left\{x \in \mathbb{R}^{n}:|x| \leq 2^{k}\right\}, A_{k}=$ $B_{k} / B_{k-1}$, and $\chi_{k}=\chi_{A_{k}}$ for $k \in \mathbb{Z}$.

Definition 6 . Let $w$ be a weight on $\mathbb{R}^{n}, \lambda \in[0, \infty), q \in(0, \infty)$, $p(\cdot) \in \mathscr{P}\left(\mathbb{R}^{n}\right)$ and $\alpha(\cdot): \mathbb{R}^{n} \longrightarrow \mathbb{R}$ with $\alpha(\cdot) \in L^{\infty}\left(\mathbb{R}^{n}\right)$. The space $M \dot{K}_{q, p(\cdot)}^{\alpha(\cdot), \lambda}(w)$ is the set of all measurable functions which is given by

$$
M \dot{K}_{q, p(\cdot)}^{\alpha(\cdot), \lambda}(w)=\left\{f \in L_{l o c}^{p(\cdot)}\left(\mathbb{R}^{n} /\{0\}, w\right):\|f\|_{M \dot{K}_{q, p(\cdot)}^{\alpha(\cdot), \lambda}(w)}<\infty\right\},
$$

where

$$
\|f\|_{M \dot{K}_{q \cdot p(\cdot)}^{\alpha(\cdot), \lambda}(w)}=\sup _{k_{0} \in Z} 2^{-k_{0} \lambda}\left(\sum_{k=-\infty}^{k_{0}} 2^{k \alpha(\cdot) q}\left\|f \chi_{k}\right\|_{L^{p(\cdot)}(w)}^{q}\right)^{1 / q} .
$$

Obviously, $M \dot{K}_{q, p(\cdot)}^{\alpha(\cdot), 0}(w)=\dot{K}_{q, p(\cdot)}^{\alpha(\cdot)}(w)$ is the weighted Herz space with variable exponent (see [30]). Here, it is important to refer to some of the pioneering studies of the Herz space with constant exponents made in $[45,46]$.

\section{Some Useful Lemmas}

We start this section with some useful lemmas that will be helpful in proving our main results.

Lemma 7 (see [47]). If $X$ is Banach function space, then

(i) The associated space $X^{\prime}$ is also Banach function space

(ii) $\|\cdot\|_{\left(X^{\prime}\right)^{\prime}}$ and $\|\cdot\|_{X}$ are equivalent

(iii) If $g \in X$ and $f \in X^{\prime}$, then

$$
\int_{\mathbb{R}^{n}}|f(x) g(x)| \leq\|g\|_{X}\|f\|_{X^{\prime}}
$$

is the generalized Hölder inequality.

Lemma 8 (see [39]). Suppose $X$ is a Banach function space. Then, we have that for all balls $B$,

$$
1 \leq \frac{1}{|B|}\left\|\chi_{B}\right\|_{X}\left\|\chi_{B}\right\|_{X^{\prime}}
$$

Lemma 9 (see $[28,39]$ ). Let $X$ be a Banach function space. Suppose that the Hardy Littlewood maximal operator $M$ is weakly bounded on $X$; that is,

$$
\left\|\chi_{\left\{M_{f}>\sigma\right\}}\right\| X \lesssim \sigma^{-1}\|f\|_{X}
$$

is true for $\sigma>0$ and for all $f \in X$. Then, we have

$$
\sup _{\text {B:ball }} \frac{1}{|B|}\left\|\chi_{B}\right\|_{X}\left\|\chi_{B}\right\|_{X^{\prime}}<\infty \text {. }
$$

Lemma 10 (see $[39,48]$ ).

(1) $X\left(\mathbb{R}^{n}, W\right)$ is Banach function space equipped with the norm

$$
\|f\|_{X\left(\mathbb{R}^{n}, W\right)}=\|f w\|_{X}
$$

where

$$
X\left(\mathbb{R}^{n}, W\right)=\{f \in M: f W \in X:\}
$$

(2) The associate space $X^{\prime}\left(\mathbb{R}^{n}, W^{-1}\right)$ is also a Banach function space

Lemma 11 (see [39]). Let $X$ be a Banach function space. Assume that $M$ is bounded on $X^{\prime}$, then there exists a constant $\delta \in(0,1)$ for all $B \subset \mathbb{R}^{n}$ and $E \subset B$,

$$
\frac{\left\|\chi_{E}\right\|_{X}}{\left\|\chi_{B}\right\|_{X}} \lesssim\left(\frac{|E|}{|B|}\right)^{\delta}
$$

The paper [16] shows that $L^{p(\cdot)}\left(\mathbb{R}^{n}\right)$ is a Banach function space and the associated space $L^{p^{\prime}(\cdot)}\left(R^{n}\right)$ with equivalent norm. Remark 12. Let $p(\cdot) \in \mathscr{P}\left(\mathbb{R}^{n}\right)$, and by comparing the Lebesgue space $L^{p(\cdot)}\left(w^{p(\cdot)}\right)$ and $L^{p^{\prime}(\cdot)}\left(w^{-p^{\prime}(\cdot)}\right)$ with the definition of $X\left(\mathbb{R}^{n}, W\right)$, we have

(1) If we take $W=w$ and $X=L^{p(\cdot)}\left(\mathbb{R}^{n}\right)$, then we get $L^{p(\cdot)}\left(\mathbb{R}^{n}, w\right)=L^{p(\cdot)}\left(w^{p(\cdot)}\right)$

(2) If we consider $W=w^{-1}$ and $X=L^{p^{\prime}(\cdot)}\left(\mathbb{R}^{n}\right)$, then we have $L^{p^{\prime}(\cdot)}\left(w^{-p^{\prime}(\cdot)}\right)=L^{p^{\prime}(\cdot)}\left(\mathbb{R}^{n}, w^{-1}\right)$

By virtue of Lemma 10 , we get $\left(L^{p(\cdot)}\left(\mathbb{R}^{n}, w\right)\right)^{\prime}=$ $\left(L^{p(\cdot)}\left(w^{p(\cdot)}\right)\right)^{\prime}=L^{p^{\prime}(\cdot)}\left(w^{-p^{\prime}(\cdot)}\right)=L^{p^{\prime}(\cdot)}\left(\mathbb{R}^{n} \cdot w^{-1}\right)$. Next, in view of Lemma 11 and Remark 12, we have the following Lemma.

Lemma 13 (see [41]). Let $p(\cdot) \in \mathscr{P}\left(R^{n}\right) \cap \mathscr{C} \log \left(\mathbb{R}^{n}\right)$ be a Log Hölder continuous function both at infinity and at origin, if $w^{p 2}(\cdot) \in A_{p 2(\cdot)}$ implies $w^{-p_{2}{ }^{\prime}} \in A_{p_{2}^{\prime}(\cdot)}$. Thus, the Hardy Littlewood operator is bounded on $L^{p_{2}{ }^{\prime}(\bullet)}\left(w^{p_{2}{ }^{\prime}(\bullet)}\right)$, and there exist constants $\delta_{1}, \delta_{2} \in(0,1)$ such that

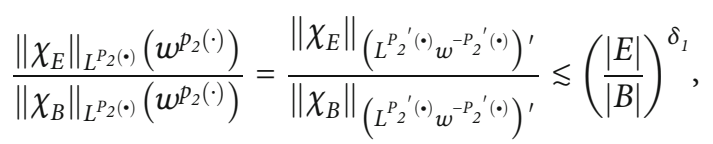

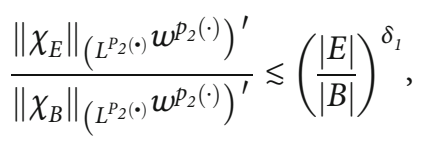

for all balls $B$ and all measurable sets $E \subset B$. 
Lemma 14 (see [39]). Let $p_{1}(\cdot) \in \mathscr{P}\left(\mathbb{R}^{n}\right) \cap \mathscr{C}^{\log }\left(R^{n}\right)$ and 0 $<\beta<n / p_{1+}$ and $1 / p_{2}(\cdot)=1 / p_{1}(\bullet)-\beta / n$. If $\in A\left(p_{1}(\cdot), p_{2}(\cdot)\right)$, then $I^{\beta}$ is bounded from $L^{p_{1}(\cdot)}\left(w^{P_{1}(\cdot)}\right)$ to $L^{p_{2}(\cdot)}\left(w^{P_{2}(\cdot)}\right)$.

\section{Main Results and their Proofs}

Definition 15. Let $f \in L_{l o c}^{1}(R n)$ and set

$$
\|b\|_{B M O}=\sup _{B} \int_{B}\left|b(x)-b_{B}\right| d x,
$$

where the supremum is taken all over the balls $B \in \mathbb{R}^{n}$ and $b_{B}=|B|^{-1} \int_{B} b(y) d y$. The function $b$ is a bounded mean oscillation if $\|b\|_{B M O}<\infty$ and $B M O\left(\mathbb{R}^{n}\right)$ consist of all $f$ $\in L_{l o c}^{1}\left(\mathbb{R}^{n}\right)$ with $B M O\left(\mathbb{R}^{n}\right)<\infty$. For a comprehensive review of the $B M O$ space, we suggest the reader to follow the books $[49,50]$.

Lemma 16. Let $q(\cdot) \in \mathscr{P}\left(\mathbb{R}^{n}\right)$ and $w$ be an $A_{q(\cdot)}$ weight. Then, for all $b \in B M O$ and all $l, i \in \mathbb{Z}$ with $l>i$, we have

$$
\begin{aligned}
& \|b\|_{B M O} \sim \sup _{B: B a l l} \frac{1}{\left\|\chi_{B}\right\|_{L^{q(\cdot)}}\left(w^{q(\cdot)}\right)}\left\|\left(b-b_{B}\right) \chi_{B}\right\|_{L^{q(\cdot)}\left(w^{q(\cdot)}\right)}, \\
& \left\|\left(b-b_{B_{i}}\right) \chi_{B_{l}}\right\|_{L^{q(\cdot)}\left(w^{q(\cdot)}\right)} \leq C(l-i)\|b\|_{B M O}\left\|\chi_{B_{l}}\right\|_{L^{q(\cdot)}\left(w^{q(\cdot)}\right)} .
\end{aligned}
$$

Proof. First part of this lemma is a consequence of [[41], Theorem 18]. Next, we will prove (28), for all $l, i \in \mathbb{Z}$ with $l>i$

$$
\begin{aligned}
& \left\|\left(b-b_{B_{i}}\right) \chi_{B_{l}}\right\|_{L^{q(\cdot)}}\left(w^{q(\cdot)}\right) \\
& \quad \leq C\left\|\left(\left|b-b_{B_{l}}\right|+\left|b-b_{B_{l}}\right|\right) \chi_{B_{l}}\right\|_{L^{q(\cdot)}\left(w^{q(\cdot)}\right)} \\
& \quad \leq C\left\{\left\|\left(b-b_{B_{l}}\right) \chi_{B_{l}}\right\|_{\left.L^{q(\cdot)\left(w^{q(\cdot)}\right.}\right)}+\left\|\left(b_{B l}-b_{B_{i}}\right) \chi_{B_{l}}\right\|_{\left.L^{q(\cdot)}\left(w^{q(\cdot)}\right)\right\} .}\right.
\end{aligned}
$$

In the view of (27), we have

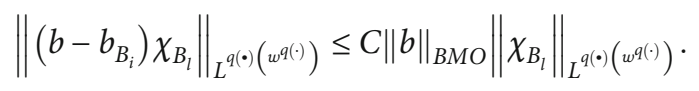

Also, it is easy to see that

$$
\begin{aligned}
\left|b_{B l}-b_{B_{i}}\right| & \leq \sum_{n=1}^{l-1}\left|b_{n+1}-b_{n}\right| \\
& \leq \sum_{n=1}^{l-1} \frac{1}{\left|B_{n}\right|} \int_{B_{n}}\left|b_{n+1}-b(x)\right| d x \\
& \leq C \sum_{n=1}^{l-1} \frac{1}{\left|B_{n+1}\right|} \int_{B_{n}}\left|b_{n+1}-b(x)\right| d x \\
& =C(l-i)\|b\|_{B M O\left(\mathbb{R}^{n}\right)^{.}}
\end{aligned}
$$

Combining (29), (30), and (31), we get (28).
Proposition 17. Let $q(\cdot) \in \mathscr{P}\left(\mathbb{R}^{n}\right), 0<p<\infty$, and $0 \leq \lambda<\infty$. If $\alpha(\cdot) \in L^{\infty}\left(\mathbb{R}^{n}\right) \cap \mathscr{C}^{\log }\left(\mathbb{R}^{n}\right)$, then

$$
\begin{aligned}
& \|f\|_{M \dot{K}_{p, q(\cdot)}^{\alpha(\cdot) \lambda}\left(w^{q(\cdot)}\right)}^{p}=\sup _{k_{0} \in Z} 2^{-k_{0} \lambda p} \sum_{j=-\infty}^{k_{0}} 2^{j \alpha(\cdot) p}\left\|f \chi_{j}\right\|_{L^{q(\cdot)}}^{p}\left(w^{q(\cdot)}\right)
\end{aligned}
$$

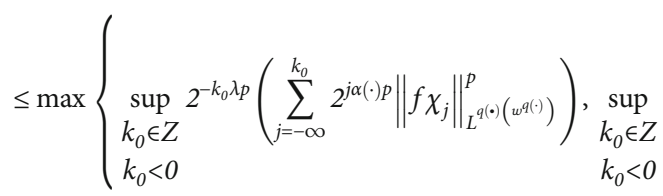

$$
\begin{aligned}
& \cdot\left(2^{-k_{0} \lambda p}\left(\sum_{j=-\infty}^{-1} 2^{j \alpha(\cdot) p}\left\|f \chi_{j}\right\|_{L^{q(\cdot)\left(w^{q(\cdot)}\right)}}^{p}\right)\right. \\
& \left.\left.+2^{-k_{0} \lambda p}\left(\sum_{j=0}^{k_{0}} 2^{j \alpha(\infty)) p}\left\|f \chi_{j}\right\|_{L^{q(\cdot)\left(w^{q(\cdot)}\right)}}^{p}\right)\right)\right\} \text {. }
\end{aligned}
$$

Proof. The proof is similar to the proof of Proposition 17 in [44]. So, we omit the details.

Theorem 18. Let $0<p_{1} \leq p_{2}<\infty, q_{2}(\cdot) \in \mathscr{P}\left(\mathbb{R}^{n}\right) \cap \mathscr{C}^{\log }\left(\mathbb{R}^{n}\right)$, and $q_{1}(\cdot)$ be such that $1 / q_{1}(\cdot)=1 / q_{2}(\bullet)-\beta / n$..

Also, let $w^{q 2}(\cdot) \in A_{1}, b \in B M O\left(\mathbb{R}^{n}\right), \lambda>0$, and $\alpha(\cdot) \in L^{\infty}$ $\left(\mathbb{R}^{n}\right) \cap \mathscr{C}^{\log }\left(\mathbb{R}^{n}\right)$ be log Hölder continuous at the origin, with $\alpha(0) \leq \alpha(\infty)<\lambda+n \delta_{2}-\beta$, where $0<\delta_{2}<1$, then

$$
\left\|\left[b, H_{\beta}\right] f\right\|_{M \dot{K}_{p_{2}, q_{2}(\cdot)}^{\alpha(\cdot),}\left(w^{q_{2}(\cdot)}\right)} \leq C\|b\|_{B M O}\|f\|_{M \dot{K}_{p_{1}, q_{1}(\cdot)}^{\alpha(\cdot),}\left(w^{q_{2}(\cdot)}\right)} .
$$

Proof. For any $f \in M \dot{K}_{p_{1}, q_{1}(\cdot)}^{\alpha(\cdot),}\left(w^{q_{1}(\cdot)}\right)$, if we denote $f_{l}=f$. $\chi_{l}=f \cdot \chi_{A_{l}}$, and for each $l \in \mathbb{Z}$,

$$
f(x)=\sum_{1=-\infty}^{\infty} f(x) \cdot \chi_{l}(x)=\sum_{1=-\infty}^{\infty} f_{l}(x)
$$

then it is not difficult to see that

$$
\begin{aligned}
\left|\left[b, H_{\beta}\right] f(x) \chi_{j}(x)\right| \leq & \frac{1}{|x|^{n-\beta}} \int_{B_{j}}|(b(x)-b(y)) f(y)| d y \cdot \chi_{j}(x) \\
\leq & 2^{-j(n-\beta)} \sum_{l=-\infty}^{j} \int_{B_{j}}|(b(x)-b(y)) f(y)| d y \cdot \chi_{j}(x) \\
\leq & 2^{-j(n-\beta)} \sum_{l=-\infty}^{j} \int_{B_{j}}\left|\left(b(x)-b_{B_{l}}\right) f(y)\right| d y \cdot \chi_{j}(x) \\
& +2^{-j(n-\beta)} \sum_{l=-\infty}^{j} \int_{B_{j}}\left|\left(b(x)-b_{B_{l}}\right) f(y)\right| d y \cdot \chi_{j}(x) \\
= & E_{1}+E_{2} .
\end{aligned}
$$


The generalized Hölder inequality (Lemma 7) yields the following inequality for $E_{1}$ :

$$
\begin{gathered}
E_{1}=2^{-j(n-\beta)} \sum_{l=-\infty}^{j} \int_{B_{l}}\left|\left(b(x)-b_{B_{l}}\right) f(y)\right| d y \cdot \chi_{j}(x) \\
\leq 2^{-j(n-\beta)} \sum_{l=-\infty}^{j}\left|\left(b(x)-b_{B_{l}}\right)\right| \cdot \chi_{j}(x)\left\|f_{l}\right\|_{L^{q_{1}} \cdot(\cdot)}\left(w^{q_{1} \cdot(\cdot)}\right) \\
\quad \times\left\|\chi_{B_{l}}\right\|_{\left.\left(L^{q_{1} \cdot(\cdot)}\left(w^{q_{1} \cdot} \cdot\right)\right)\right)^{\prime}}
\end{gathered}
$$

Applying the norm on both sides and using Lemma 16 , we get

$$
\begin{aligned}
& \left\|E_{1}\right\|_{\left(L^{q_{2} \cdot \cdot}\left(w^{q_{2}(\cdot)}\right)\right)} \leq 2^{-j(n-\beta)} \sum_{l=-\infty}^{j}\left\|\left(b(x)-b_{B_{l}} \cdot \chi_{B_{j}}\right)\right\|_{\left(L^{q_{2} \cdot \cdot}\left(w^{q_{2} \cdot \cdot}\right)\right)} \\
& \cdot\left\|f_{l}\right\|_{L^{q_{1} \cdot} \cdot\left(w^{q_{1}(\cdot)}\right)}\left\|\chi_{B_{l}}\right\|_{\left(L^{q_{1}(\cdot)}\left(w^{q_{1}(\cdot)}\right)\right)^{\prime}} \\
& \leq 2^{-j(n-\beta)} \sum_{l=-\infty}^{j}(j-l)\|b\|_{B M O}\left\|\chi_{B_{l}}\right\|_{\left(L^{q_{2} \cdot \cdot}\left(w^{q_{2}(\cdot)}\right)\right)^{\prime}} \\
& \cdot\left\|f_{l}\right\|_{L^{q_{1} \cdot \cdot}\left(w^{q_{1} \cdot} \cdot\right)}\left\|\chi_{B_{l}}\right\|_{\left(L^{q_{1} \cdot \cdot}\left(w^{q_{1} \cdot(\cdot)}\right)\right)^{\prime}} \cdot
\end{aligned}
$$

Now, we turn to estimate $E_{2}$. For this, we have

$$
\begin{aligned}
E_{2} \leq & 2^{-j(n-\beta)} \sum_{l=-\infty}^{j}\left\|\left(b(y)-b_{B_{l}} \cdot \chi_{l}\right)\right\|_{\left(L^{q_{1} \cdot \cdot}\left(w^{q_{1} \cdot(\cdot)}\right)\right)^{\prime}} \\
& \cdot\left\|f_{l}\right\|_{\left.L^{q_{1}} \cdot \cdot\right)\left(w^{\left.q_{1} \cdot \cdot\right)}\right)} \cdot \chi_{j}(x) \\
\leq & 2^{-j(n-\beta)} \sum_{l=-\infty}^{j}\left\|\left(b(y)-b_{B_{l}} \cdot \chi_{B_{l}}\right)\right\|_{\left(L^{q_{1} \cdot \cdot}\left(w^{q_{1}(\cdot)}\right)\right)^{\prime}} \\
& \cdot\left\|f_{l}\right\|_{\left.L^{q_{1}} \cdot \cdot\right)\left(w^{q_{1} \cdot} \cdot\right)} \cdot \chi_{j}(x) .
\end{aligned}
$$

Similar to the estimation for $E_{1}$, we take the norm on both sides of above inequality and use Lemma 16 to obtain

$$
\begin{aligned}
\left\|E_{2}\right\|_{L^{q_{2}(\cdot)}\left(w^{q_{2}(\cdot)}\right)} \leq 2^{-j(n-\beta)} \sum_{l=-\infty}^{j}\left\|\left(b(y)-b_{B_{l}}\right) \cdot \chi_{l}\right\|_{\left(L^{q_{1} \cdot} \cdot\left(w^{q_{1}(\cdot)}\right)\right)^{\prime}} \\
\cdot\left\|f_{l}\right\|_{L^{q_{1}(\cdot)}\left(w^{\left.q_{1} \cdot \cdot\right)}\right)} \cdot\left\|\chi_{j}\right\|_{L^{q_{2}(\cdot)}\left(w^{q_{2}(\cdot)}\right)} \\
\leq 2^{-j(n-\beta)} \sum_{l=-\infty}^{j}\|b\|_{B M O}\left\|\chi_{B_{l}}\right\|_{\left(L^{q_{1} \cdot \cdot}\left(w^{q_{1} \cdot \cdot}\right)\right)^{\prime}} \\
\cdot\left\|f_{l}\right\|_{L^{q_{1}(\cdot)}\left(w^{q_{1}(\cdot)}\right)}\left\|\chi_{B_{j}}\right\|_{\left(L^{q_{2} \cdot}(\cdot)\left(w^{q_{2}(\cdot)}\right)\right)} \cdot
\end{aligned}
$$

Hence, from inequalities (35), (37), and (39), one has $\quad\left\|\left[b, H_{\beta}\right] f \chi_{j}\right\|_{L^{q_{2}(\cdot)}\left(w^{q_{2}(\cdot)}\right)} \leq 2^{-j(n-\beta)}\|b\|_{B M O} \sum_{l=-\infty}^{j}(j-1)$
$\left\|f_{l}\right\|_{L^{q_{1} \cdot \cdot(\cdot)}\left(w^{q_{1} \cdot(\cdot)}\right)}\left\|\chi_{B_{j}}\right\|_{\left(L^{\left.q_{2} \cdot \cdot\right)}\left(w^{\left.q_{2} \cdot \cdot\right)}\right)\right)}\left\|\chi_{B_{l}}\right\|_{\left(L^{q_{1} \cdot(\cdot)}\left(w^{\left.q_{1} \cdot(\cdot)\right)}\right)\right.}$, which by virtue of Lemma 9 reduces to

$$
\begin{aligned}
\left\|\left[b, H_{\beta}\right] f \chi_{j}\right\|_{L^{q_{2}(\cdot)}\left(w^{q_{2}(\cdot)}\right)} \leq 2^{j \beta}\|b\|_{B M O} \sum_{l=-\infty}^{j}(j-l)\left\|f_{l}\right\|_{L^{q_{1}(\cdot)}\left(w^{q_{1}(\cdot)}\right)} \\
\cdot\left\|\chi_{B_{l} l}\right\|_{\left(L^{q_{1}(\cdot)}\left(w^{q_{1}(\cdot)}\right)\right)^{\prime}}\left\|\chi_{B_{j}}\right\|_{\left(L^{q_{2}(\cdot)}\left(w^{q_{2}(\cdot)}\right)\right)^{\prime}}^{-1}
\end{aligned}
$$

Now using Lemma 13, we learn

$$
\begin{aligned}
& \left\|\left[b, H_{\beta}\right] f \chi_{j}\right\|_{L^{q_{2}(\cdot)}\left(w^{q_{2}(\cdot)}\right)} \\
& \leq 2^{j \beta}\|b\|_{B M O} \sum_{l=-\infty}^{j}(j-l)\left\|f_{l}\right\|_{L^{q_{1}(\cdot)}\left(w^{q_{1}(\cdot)}\right)}
\end{aligned}
$$

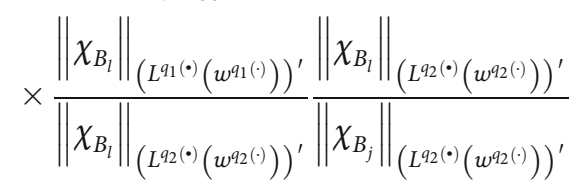

$$
\begin{aligned}
& \leq 2^{j \beta}\|b\|_{B M O} \sum_{l=-\infty}^{j}(j-l) 2^{(l-j) n \delta_{2}}\|b\|_{B M O}\left\|f_{l}\right\|_{L^{q_{1} \cdot(\cdot)}\left(w^{q_{1}(\cdot)}\right)} \\
& \times \frac{\left\|\chi_{B_{l}}\right\|_{\left(L^{q_{1} \cdot \cdot}\left(w^{q_{1} \cdot \cdot}\right)\right)^{\prime}}}{\left\|\chi_{B_{l}}\right\|_{\left(L^{q_{2} \cdot \cdot}\left(w^{q_{2} \cdot \cdot}\right)\right)^{\prime}}}
\end{aligned}
$$

In the definition of the fraction integral $I_{\beta}$, we replace $f$ by $\chi_{B l}$ to obtain

$$
I_{\beta}\left(\chi_{B_{l}}\right)(x) \geq C 2^{l \beta} \chi_{B_{l}}(x)
$$

from which we infer that

$$
\chi_{B_{l}}(x) \leq C 2^{-l \beta} I \beta\left(\chi_{B_{l}}\right)(x)
$$

Taking the norm on both sides and using Lemmas 14 and 9 , respectively, we get

$$
\begin{aligned}
\left\|\chi_{B_{l}}\right\|_{L^{q_{2}(\cdot)}\left(w^{q_{2}(\cdot)}\right)} \leq C 2^{l \beta}\left\|I_{\beta}\left(\chi_{B_{l}}\right)\right\|_{L^{q_{2}(\cdot)}\left(w^{q_{2}(\cdot)}\right)} \\
\leq C 2^{l \beta}\left\|\chi_{B_{l}}\right\|_{L^{q_{1}(\cdot)}\left(w^{q_{1}(\cdot)}\right)} \\
\leq C 2^{l(n-\beta)}\left\|\chi_{B_{l}}\right\|_{\left(L^{q_{1} \cdot \cdot}\left(w^{q_{1}(\cdot)}\right)\right)^{\prime}}^{-1}
\end{aligned}
$$


In view of Lemmas 8 and 9, the use of (44) into (41) results in the following inequality:

$$
\begin{aligned}
\left\|\left[b, H_{\beta}\right] f \chi_{j}\right\|_{L^{q_{2}(\cdot)}\left(w^{q_{2}(\cdot)}\right)} & \\
\leq & C b\left\|_{B M O} \sum_{l=-\infty}^{j} 2^{l(n-\beta)} 2^{j \beta}(j-l) 2^{(l-j) n \delta_{2}}\right\| f_{l} \|_{L^{q_{1}(\cdot)}\left(w^{q_{1}(\cdot)}\right)} \\
& \times\left(\left\|\chi_{l}\right\|_{\left(L^{q_{2}(\cdot)}\left(w^{q_{2}(\cdot)}\right)\right)}\left\|\chi_{l}\right\|_{\left(L^{q_{2}(\cdot)}\left(w^{q_{2}(\cdot)}\right)\right)^{\prime}}\right)^{-1} \\
\leq & C\|b\|_{B M O} \sum_{l=-\infty}^{j} 2^{(j-l)\left(\beta-n \delta_{2}\right)}(j-l)\left\|f_{l}\right\|_{L^{q_{1}(\cdot)}\left(w^{q_{1}(\cdot)}\right)} \\
& \times\left(2^{-\ln }\left\|\chi_{l}\right\|_{\left(L^{q_{2}(\cdot)}\left(w^{q_{2}(\cdot)}\right)\right)}\left\|\chi_{l}\right\|_{\left(L^{q_{2}(\cdot)}\left(w^{q_{2}(\cdot)}\right)\right)^{\prime}}\right)^{-1} \\
\leq & C\|b\|_{B M O} \sum_{l=-\infty}^{j} 2^{(j-l)\left(\beta-n \delta_{2}\right)}(j-l)\left\|f_{l}\right\|_{L^{q_{1}(\cdot)}\left(w^{q_{1}(\cdot)}\right)} .
\end{aligned}
$$

Now, by virtue of the condition $p_{1} \leq p_{2}$ and Proposition 17 , we have

$$
\begin{aligned}
& \left\|\left[b, H_{\beta}\right] f \chi_{j}\right\|_{M \dot{K}_{p_{2} q_{2}(\cdot)}^{\alpha(\cdot),}\left(w^{q_{2}(\cdot)}\right)}^{p_{1}} \\
& \leq \max \left\{\begin{array}{l}
\sup _{k_{0} \in Z} 2^{-k_{0} \lambda p_{1}}\left(\sum_{j=-\infty}^{k_{0}} 2^{j \alpha(0) p_{1}}\left\|\left[b, H_{\beta}\right] f \chi_{j}\right\|_{L^{q_{2}(\cdot)}\left(w^{\left.q_{2} \cdot \cdot\right)}\right)}^{p_{1}}\right), \sup _{k_{0} \in Z} \\
k_{0} \geq 0
\end{array}\right. \\
& \times\left(2^{-k_{0} \lambda p_{1}}\left(\sum_{j=-\infty}^{k_{0}} 2^{j \alpha(0) p_{1}}\left\|\left[b, H_{\beta}\right] f \chi_{j}\right\|_{L^{q_{2}(\cdot)}\left(w^{q_{2}(\cdot)}\right)}^{p_{1}}\right)\right. \\
& \left.\left.+2^{-k_{0} \lambda p_{1}}\left(\sum_{j=0}^{k_{0}} 2^{j \alpha(\infty) p_{1}}\left\|\left[b, H_{\beta}\right] f \chi_{j}\right\|_{L^{q_{2}(\cdot)}\left(w^{q_{2}(\cdot)}\right)}^{p_{1}}\right)\right)\right\} \\
& =\max \left\{X_{1}, X_{2}, X_{3}\right\},
\end{aligned}
$$

where

$$
\begin{aligned}
& X_{1}= \sup _{\substack{k_{0} \in Z \\
k_{0}<0}} 2^{-k_{0} \lambda p_{1}}\left(\sum_{j=-\infty}^{k_{0}} 2^{j \alpha(0) p_{1}}\left\|\left[b, H_{\beta}\right] f \chi_{j}\right\|_{L^{q_{2}(\cdot)}\left(w^{q_{2}(\cdot)}\right)}^{p_{1}}\right), \\
& X_{2}= \sup _{\substack{k_{0} \in Z \\
k_{0} \geq 0}} 2^{-k_{0} \lambda p_{1}}\left(\sum_{j=-\infty}^{-1} 2^{j \alpha(0) p_{1}}\left\|\left[b, H_{\beta}\right] f \chi_{j}\right\|_{L^{q_{2}(\cdot)}\left(w^{q_{2}(\cdot)}\right)}^{p_{1}}\right), \\
& X_{3}=\sup _{\substack{k_{0} \in Z \\
k_{0} \geq 0}} 2^{-k_{0} \lambda p_{1}}\left(\sum_{j=0}^{k_{0}} 2^{j \alpha(0) p_{1}}\left\|\left[b, H_{\beta}\right] f \chi_{j}\right\|_{L^{q_{2}(\cdot)}\left(w^{q_{2}(\cdot)}\right)}^{p_{1}}\right) .
\end{aligned}
$$

To estimate $X_{1}, X_{2}$, and $X_{3}$, we make use of the conditions on $\alpha(\cdot)$, such that for $l<0$, we have

$$
\begin{aligned}
\left\|f_{l}\right\|_{L^{q_{1}(\cdot)}\left(w^{q_{1}(\cdot)}\right)} & =2^{-l \alpha(0)}\left(2^{j \alpha(0) p_{1}}\left\|f_{l}\right\|_{L^{q_{1}(\cdot)}\left(w^{q_{1}(\cdot)}\right)}^{p_{p}}\right)^{\frac{1}{p_{1}}} \\
& \leq 2^{-l \alpha(0)}\left(\sum_{1=-\infty}^{l} 2^{i \alpha(0) p_{1}}\left\|f_{i}\right\|_{L^{q_{1}}(\cdot)}^{p_{1}}\left(w^{q_{1}(\cdot)}\right)\right)^{\frac{1}{p_{1}}} \\
& \leq 2^{l(\lambda-\alpha(0))} 2^{-l \lambda}\left(\sum_{1=-\infty}^{l} 2^{i \alpha(\cdot) p_{1}}\left\|f_{i}\right\|_{L^{q_{1}(\cdot)}\left(w^{q_{1}(\cdot)}\right)}^{p_{p}}\right)^{\frac{1}{p_{1}}} \\
& \leq C 2^{l(\lambda-\alpha(0))}\|f\|_{M \dot{K}_{p_{1}, q_{1}(\cdot),}^{\alpha(\cdot)}\left(w^{q_{1}(\cdot)}\right)}
\end{aligned}
$$

and for $l \geq 0$, we obtain

$$
\begin{aligned}
\left\|f_{l}\right\|_{L^{q_{1}(\cdot)}\left(w^{q_{1}(\cdot)}\right)} & =2^{-l \alpha(\infty)}\left(2^{l \alpha(\infty) p_{1}}\left\|f_{l}\right\|_{L^{q_{1}(\cdot)}\left(w^{q_{1}(\cdot)}\right)}^{p_{1}}\right)^{\frac{1}{p_{1}}} \\
& \leq 2^{-l \alpha(\infty)}\left(\sum_{1=-\infty}^{l} 2^{i \alpha(\infty) p_{1}}\left\|f_{i}\right\|_{L^{q_{1}(\cdot)}\left(w^{q_{1}(\cdot)}\right)}^{p_{1}}\right)^{\frac{1}{p_{1}}} \\
& \leq 2^{l(\lambda-\alpha(\infty))} 2^{-l \lambda}\left(\sum_{1=-\infty}^{l} 2^{i \alpha(\cdot) p_{1}}\left\|f_{i}\right\|_{L^{q_{1}(\cdot)}\left(w^{q_{1}(\cdot)}\right)}^{p_{1}}\right)^{\frac{1}{p_{1}}} \\
& \leq C 2^{l(\lambda-\alpha(\infty))}\|f\|_{M \dot{K}_{p_{1}, q_{1}(\cdot),}^{\alpha(\cdot)}\left(w^{q_{1}(\cdot)}\right)} \cdot
\end{aligned}
$$

In order to estimate $X_{1}$, we need to use $\alpha(0) \leq \alpha(\infty)<n$ $\delta_{2}+\lambda-\beta$.

$$
\begin{aligned}
& X_{1} \leq \sup _{k_{0} \in Z} 2^{-k_{0} \lambda p_{1}} \sum_{j=-\infty}^{k_{0}} 2^{j \alpha(0) p_{1}} \\
& k_{0}<0 \\
& \cdot\left(\sum_{l=-\infty}^{j}(j-l) 2^{(j-l)\left(\beta-n \delta_{2}\right)}\|b\|_{B M O}\left\|f_{l}\right\|_{L^{q_{1}(\cdot)}\left(w^{q_{1}(\cdot)}\right)}\right)^{p_{1}} \\
& \leq C \sup _{k_{0} \in Z} 2^{-k_{0} \lambda p_{1}} \sum_{j=-\infty}^{k_{0}} 2^{j \alpha(0) p_{1}} \\
& k_{0}<0 \\
& \cdot\left(\sum_{l=-\infty}^{\mathrm{j}}(j-l) 2^{(j-l)\left(\beta-n \delta_{2}\right)} 2^{l(\lambda-\alpha(0))}\|b\|_{B M O}\|f\|_{M \dot{K}_{p_{1}, q_{1}(\cdot)}^{\alpha(\cdot),}\left(w^{q_{1}(\cdot)}\right)}\right)^{p_{1}} \\
& \leq C \sup _{\substack{k_{0} \in Z \\
k_{0}<0}} 2^{-k_{0} \lambda p_{1}} \sum_{j=-\infty}^{k_{0}} 2^{j \alpha(0) p_{1}}\left(\sum_{l=-\infty}^{j}(j-l) 2^{(j-l)\left(\beta-n \delta_{2}\right)} 2^{l(\lambda-\alpha(0))}\right)^{p_{1}} \\
& k_{0}<0 \\
& \cdot\|b\|_{B M O}^{p_{1}}\|f\|_{M \dot{K}_{p_{1}, q_{1}(\cdot)}^{\alpha(\cdot),}}^{p_{1}}\left(w^{q_{1}(\cdot)}\right)
\end{aligned}
$$




$$
\begin{aligned}
& \leq C \sup _{\substack{k_{0} \in Z \\
k_{0}<0}} 2^{-k_{0} \lambda p_{1}} \sum_{j=-\infty}^{k_{0}} 2^{j \lambda p_{1}}\left(\sum_{l=-\infty}^{j}(j-l) 2^{(l-j)\left(-\beta+n \delta_{2}-\alpha(0)+\lambda\right)}\right)^{p_{1}} \\
& \cdot\|b\|_{B M O}^{p_{1}}\|f\|_{M \dot{K}_{p_{1}, q_{1}(\cdot)}^{(\cdot) \cdot \lambda}}^{p_{1}}\left(w^{q_{1}(\cdot)}\right) \\
& \left.\leq C\|b\|_{B M O}^{p_{1}}\|f\|_{M \dot{K}_{p_{1}, q_{1}(\cdot)}^{\alpha(\cdot)}\left(w^{q_{1}(\cdot)}\right)}^{p_{1}}\right)
\end{aligned}
$$

The result of $X_{2}$ is similar to that of $X_{1}$. Next, we will estimate $X_{3}$ below

$$
\begin{aligned}
& X_{3} \leq \sup _{k_{0} \in Z} 2^{-k_{0} \lambda p_{1}} \sum_{j=-\infty}^{k_{0}} 2^{j \alpha(\infty)) p_{1}} \\
& k_{0}<0 \\
& \cdot\left(\sum_{l=-\infty}^{j}(j-l) 2^{(j-l)\left(\beta-n \delta_{2}\right)}\|b\|_{B M O}\left\|f_{l}\right\|_{L^{\left.q_{1} \cdot \cdot\right)}\left(w^{q_{1} \cdot(\cdot)}\right)}\right)^{p_{1}} \\
& \leq C \sup _{k_{0} \in Z} 2^{-k_{0} \lambda p_{1}} \sum_{j=-\infty}^{k_{0}} 2^{j \alpha(\infty) p_{1}} \\
& k_{0}<0 \\
& \cdot\left(\sum_{l=-\infty}^{j}(j-l) 2^{(j-l)\left(\beta-n \delta_{2}\right)} 2^{l(\lambda-\alpha(\infty))}\|b\|_{B M O}\|f\|_{M \dot{K}_{p_{1}, q_{1}(\cdot)}^{\alpha(\cdot),}\left(w^{q_{2}(\cdot)}\right)}\right)^{p_{1}} \\
& \leq C \sup _{k_{0} \in Z} 2^{-k_{0} \lambda p_{1}} \sum_{j=-\infty}^{k_{0}} 2^{j \alpha(\infty) p_{1}}\left(\sum_{l=-\infty}^{j}(j-l) 2^{(j-l)\left(\beta-n \delta_{2}\right)} 2^{l(\lambda-\alpha(\infty)))}\right)^{p_{1}} \\
& k_{0}<0 \\
& \cdot\|b\|_{B M O}^{p_{1}}\|f\|_{M \dot{K}_{p_{1} q_{1}(\cdot)}^{\alpha(\cdot) \lambda}}^{p_{1}}\left(w^{q_{2}(\cdot)}\right) \\
& \leq C \sup _{k_{0} \in Z} 2^{-k_{0} \lambda p_{1}} \sum_{j=-\infty}^{k_{0}} 2^{j \lambda p_{1}}\left(\sum_{l=-\infty}^{j}(j-l) 2^{(l-j)\left(-\beta+n \delta_{2}-\alpha(\infty)+\lambda\right)}\right)^{p_{1}} \\
& k_{0}<0 \\
& \left.\cdot\|b\|_{B M O}^{p_{1}}\|f\|_{M \dot{K}_{p_{1} q_{1}(\cdot)}^{\alpha(j),}}^{p_{1}} w^{q_{2}(\cdot)}\right)
\end{aligned}
$$

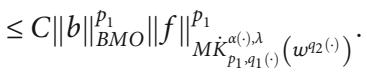

Finally, we combine the estimates for $X_{i}(i=1,2,3)$, to have the desired result.

Theorem 19. $\operatorname{Let}_{1}, p_{2}, q_{1}(\cdot), q_{2}(\cdot), \beta, \alpha(\cdot)$ and $w$ be as in Theorem 18. In addition, if $\lambda-n \delta_{1}<\alpha(0) \leq \alpha(\infty)$, where 1 $<\delta_{1}<0$, then

$$
\left\|\left[b, H_{\beta}^{*}\right] f\right\|_{M \dot{K}_{p_{2}, q_{2}(\cdot)}^{\alpha(\cdot),}\left(w^{q_{2}(\cdot)}\right)} \leq C\|b\|_{B M O}\|f\|_{M \dot{K}_{p_{1}, q_{1}(\cdot)}^{\alpha(\cdot),}\left(w^{q_{2}(\cdot)}\right)} .
$$

Proof. We write

$$
\begin{aligned}
{\left[b, H_{\beta}^{*}\right] f(x) \chi_{j}(x) } & \leq \int_{\mathbb{R}^{n \mid B_{j}}}|y|^{\beta-n}|(b(x)-b(y)) f(y)| d y \cdot \chi_{j}(x) \\
& \leq \sum_{l=j+1}^{\infty} \int_{B_{l}}|y|^{\beta-n}|(b(x)-b(y)) f(y)| d y \cdot \chi_{j}(x) \\
& \leq \sum_{l=j+1}^{\infty} \int_{B_{l}}|y|^{\beta-n}\left|b(x)-b_{B_{l}}\right| f(y) d y \cdot \chi_{j}(x)+ \\
& \leq \sum_{l=j+1}^{\infty} \int_{B_{l}}|y|^{\beta-n}\left|\left(b(y)-b_{B_{l}}\right) f(y)\right| d y \cdot \chi_{j}(x) \\
& =F_{1}+F_{2} .
\end{aligned}
$$

We estimate $F_{1}$ and $F_{2}$ separately. A use of generalized inequality results in the following:

$$
\begin{aligned}
F_{1} \leq & C \sum_{l=j+1}^{\infty} 2^{-l(n-\beta)} \int_{B_{l}}\left|\left(b(x)-b_{B_{l}}\right) f(y)\right| d y \cdot \chi_{j}(x) \\
\leq & C \sum_{l=j+1}^{\infty} 2^{-l(n-\beta)}\left\|f_{i}\right\|_{L^{q_{1}(\cdot)}\left(w^{q_{1}(\cdot)}\right)}\left\|\chi_{B_{l}}\right\|_{L^{q_{1}(\cdot)}\left(w^{q_{1}(\cdot)}\right)^{\prime}} \\
& \cdot\left|b(x)-b_{B_{l}}\right| \cdot \chi_{j} .
\end{aligned}
$$

Applying the weighted Lebesgue space norm on both sides and using Lemma 16, we obtain

$$
\begin{aligned}
& \left\|F_{1}\right\|_{L^{q_{2} \cdot \cdot}\left(w^{q_{2} \cdot(\cdot)}\right)} \leq C \sum_{l=j+1}^{\infty} 2^{-l(n-\beta)}\left\|\left(b(x)-b_{B_{j}}\right) \chi_{j}\right\|_{L^{\left.q_{2} \cdot \cdot\right)}\left(w^{q_{2}(\cdot)}\right)} \\
& \cdot\left\|f_{i}\right\|_{L^{q_{1} \cdot \cdot}\left(w^{\left.q_{1} \cdot \cdot\right)}\right)}\left\|\chi_{B_{l}}\right\|_{L^{q_{1} \cdot \cdot}\left(w^{q_{1}(\cdot)}\right)^{\prime}} \\
& \leq C \sum_{l=j+1}^{\infty} 2^{-l(n-\beta)}\|b\|_{B M O}\left\|\left(b(x)-b_{B_{j}}\right) \chi_{j}\right\|_{L^{q_{2} \cdot \cdot \cdot}\left(w^{q_{2}(\cdot)}\right)} \\
& \cdot\left\|f_{i}\right\|_{L^{q_{1} \cdot} \cdot\left(w^{q_{1}(\cdot)}\right)}\left\|\chi_{B_{l}}\right\|_{L^{q_{1} \cdot(\cdot)}\left(w^{q_{1}(\cdot)}\right)^{\prime}} \text {. }
\end{aligned}
$$

Similarly,

$$
\begin{aligned}
F_{2} \leq & C \sum_{l=j+1}^{\infty} 2^{-l(n-\beta)} \int_{B_{l}}\left|\left(b(y)-b_{B_{l}}\right) f(y)\right| d y \cdot \chi_{j}(x) \\
\leq & C \sum_{l=j+1}^{\infty} 2^{-l(n-\beta)}\left\|b(y)-b_{B_{l}} \cdot \chi_{j}\right\|_{L^{q_{1}(\cdot)}\left(w^{q_{1}(\cdot)}\right)} \\
& \cdot\left\|f_{l}\right\|_{L^{q_{1} \cdot \cdot}\left(w^{q_{1}(\cdot)}\right)^{\prime}} \cdot \chi_{j}(x)(x) .
\end{aligned}
$$


In view of the weighted Lebesgue norm and Lemma 16, we get

$$
\begin{aligned}
\left\|F_{2}\right\|_{L^{q_{2}(\cdot)}\left(w^{q_{2}(\cdot)}\right) \leq} & C \sum_{l=j+1}^{\infty} 2^{-l(n-\beta)}\left\|\left(b(y)-b_{B_{l}}\right) \cdot \chi_{j}\right\|_{\left(L^{q_{1}(\cdot)}\left(w^{q_{1}(\cdot)}\right)\right)^{\prime}} \\
& \cdot\left\|f_{l}\right\|_{L^{q_{1}(\cdot)}\left(w^{q_{1}(\cdot)}\right)} \cdot\left\|\chi_{j}\right\|_{L^{q_{2}(\cdot)}\left(w^{q_{2}(\cdot)}\right)} \\
\leq & C \sum_{l=j+1}^{\infty} 2^{-l(n-\beta)}(l-j)\|b\|_{B M O}\left\|\chi_{l}\right\|_{\left(L^{q_{1}(\cdot)}\left(w^{q_{1}(\cdot)}\right)\right)^{\prime}} \\
& \cdot\left\|f_{l}\right\|_{L^{q_{1}(\cdot)}\left(w^{q_{1}(\cdot)}\right)} \cdot\left\|\chi_{j}\right\|_{L^{q_{2}(\cdot)}\left(w^{q_{2}(\cdot)}\right)} \cdot
\end{aligned}
$$

Hence, from (53), (55), and (57), we obtain

$$
\begin{aligned}
\left\|\left[b, H_{\beta}^{*}\right] f \chi_{j}\right\|_{L^{q_{2}(\cdot)}\left(w^{q_{2}(\cdot)}\right)} & \sum_{l=j+1}^{\infty} 2^{-l(n-\beta)}(l-j)\|b\|_{B M O}\left\|\chi_{j}\right\|_{L^{q_{2}(\cdot)}\left(w^{q_{2}(\cdot)}\right)} \\
& \times\left\|f_{l}\right\|_{L^{q_{1}(\cdot)}\left(w^{q_{1}(\cdot)}\right)} \cdot\left\|\chi_{B_{l}}\right\|_{\left(L^{q_{1}(\cdot)}\left(w^{q_{1}(\cdot)}\right)\right)^{\prime}} \\
\leq & \sum_{l=j+1}^{\infty} 2^{-l(n-\beta)}(l-j)\|b\|_{B M O}\left\|f_{l}\right\|_{L^{q_{1}(\cdot)}\left(w^{q_{1}(\cdot)}\right)}\left\|\chi_{j}\right\|_{L^{q_{2}(\cdot)}\left(w^{q_{2}(\cdot)}\right)} \\
& \times\left\|\chi_{B_{l}}\right\|_{\left(L^{q_{1}(\cdot)}\left(w^{q_{1}(\cdot)}\right)\right)^{\prime}}\left\|\chi_{l}\right\|_{L^{q_{2}(\cdot)}\left(w^{q_{2}(\cdot)}\right)}\left\|\chi_{l}\right\|_{L^{q_{2}(\cdot)}\left(w^{q_{2}(\cdot)}\right)} \\
\leq & \sum_{l=j+1}^{\infty} 2^{n \delta(j-1)} 2^{-l(n-\beta)}(l-j)\|b\|_{B M O}\left\|f_{1}\right\|_{L^{q_{1}(\cdot)}\left(w^{q_{1}(\cdot)}\right)}\left\|\chi_{l}\right\|_{L^{q_{2}(\cdot)}\left(w^{q_{2}(\cdot)}\right)} \cdot
\end{aligned}
$$

Using the condition of $A\left(q_{1}(\cdot), q_{2}(\cdot)\right)$ weights given in the Definition 4 , the above inequality reduces to

$$
\begin{aligned}
& \left\|\left[b, H_{\beta}^{*}\right] f \chi_{j}\right\|_{L^{q_{2}(\cdot)}\left(w^{q_{2}(\cdot)}\right)} \\
& \quad \leq \sum_{l=j+1}^{\infty} 2^{n \delta_{1}(j-l)}(l-j)\|b\|_{B M O}\left\|f_{l}\right\|_{L^{q_{1}(\cdot)}\left(w^{q_{1}(\cdot)}\right)} .
\end{aligned}
$$

Next, the condition $p_{1}<p_{2}$ and Proposition 17 help us to write

$$
\left\|\left[b, H_{\beta}^{*}\right] f \chi_{j}\right\|_{M \dot{K}_{p_{2}, q_{2}(\cdot)}^{\alpha(\cdot),}\left(w^{q_{2}(\cdot)}\right)}^{p_{1}}=\max \left\{Y_{1}, Y_{2}, Y_{3}\right\},
$$

where

$$
\begin{aligned}
& Y_{1}=\sup _{\substack{k_{0} \in Z \\
k_{0}<0}} 2^{-k_{0} \lambda p_{1}}\left(\sum_{j=-\infty}^{k_{0}} 2^{j \alpha(0) p_{1}}\left\|\left[b, H_{\beta}^{*}\right] f \cdot \chi_{j}\right\|_{L^{q_{2}(\cdot)}\left(w^{q_{2}(\cdot)}\right)}^{p_{1}}\right), \\
& Y_{2}=\sup _{\substack{k_{0} \in Z \\
k_{0} \geq 0}} 2^{-k_{0} \lambda p_{1}}\left(\sum_{j=-\infty}^{-1} 2^{j \alpha(0) p_{1}}\left\|\left[b, H_{\beta}^{*}\right] f \cdot \chi_{j}\right\|_{L^{q_{2}(\cdot)}\left(w^{q_{2}(\cdot)}\right)}^{p_{1}}\right), \\
& Y_{3}=\sup _{\substack{k_{0} \in Z \\
k_{0} \geq 0}} 2^{-k_{0} \lambda p_{1}}\left(\sum_{j=0}^{k_{0}} 2^{j \alpha(\infty) p_{1}}\left\|\left[b, H_{\beta}^{*}\right] f \cdot \chi_{j}\right\|_{L^{q_{2}(\cdot)}\left(w^{q_{2}(\cdot)}\right)}^{p_{1}}\right) .
\end{aligned}
$$

Lastly, in view of the condition $-n \delta_{1}+\lambda<\alpha(0) \leq \alpha(\infty)$, we estimate $Y_{i}, i=1,2,3$, as we estimated $X_{i}, i=1,2,3$, in Theorem 18. Hence, we finish the proof.

\section{Data Availability}

No data were used to support this study.

\section{Conflicts of Interest}

The authors declare that they have no competing interests.

\section{Acknowledgments}

The authors would like to thank the referees for careful reading of the paper andvaluable suggestions. Amjad Hussain is supported by Higher Education Commission (HEC) of Pakistan through the National Research Program for Universities (NRPU) Project No: 7098/Federal/NRPU/R\&D/HEC/2017 and the Quaid-I-Azam University Research Fund (URF) Project. The authors extend their appreciation to the Deanship of Scientific Research at King Khalid University, Abha 61413, Saudi Arabia for funding this work through research groups program under grant number R.G. P-2/29/42.

\section{References}

[1] R. C. Brown and D. B. Hinton, "A weighted Hardy's inequality and nonoscillatory differential equations," Quaestiones Mathematicae, vol. 15, no. 2, pp. 197-212, 1992.

[2] R. C. Brown and D. B. Hinton, "Some one variable weighted norm inequalities and their applications to sturm-liouville and other differential operators," International Series of Numerical Mathematics, vol. 157, pp. 61-76, 2008.

[3] Z. W. Fu, Z. G. Liu, S. Z. Lu, and H. Wong, "Characterization for commutators of n-dimensional fractional Hardy operators," Science in China (Scientia Sinica), vol. 50, no. 10, pp. 1418-1426, 2007.

[4] Z. Fu and S. Lu, "A characterization of $\lambda$-central BMO spaces," Frontiers of Mathematics in China, vol. 8, article 229238, 2013. 
[5] S. Shi and S. Lu, "Characterization of the central Campanato space via the commutator operator of Hardy type," Journal of Mathematical Analysis and Applications, vol. 429, no. 2, pp. 713-732, 2015.

[6] W. G. Faris, "Weak Lebesgue spaces and quantum mechanical binding," Duke Mathematical Journal, vol. 43, no. 2, article 365373, 1976.

[7] M. Chris and L. Grafakos, "Best constant for two nonconvolution inequalities," Mathematische Zeitschrift, vol. 123, pp. 1687-1693, 1995.

[8] G. H. Hardy, "Note on a theorem of Hilbert," Mathematische Zeitschrift, vol. 6, no. 3-4, pp. 314-317, 1920.

[9] A. G. Bliss, "An integral inequality," Journal of the London Mathematical Society, vol. s1-5, no. 1, pp. 40-46, 1930.

[10] Z. W. Fu, L. Grafakos, S. Z. Lu, and F. Y. Zhao, "Sharp bounds for m-linear Hardy and Hilbert operators," Houston Journal of Mathematics, vol. 38, no. 1, pp. 225-244, 2012.

[11] A. Hussain and G. Gao, "Some new estimates for the commutators of n-dimensional Hausdorff operator," Applied Mathematics-A Journal of Chinese Universities, vol. 29, no. 2, pp. 139-150, 2014.

[12] L.-E. Persson and S. G. Samko, "A note on the best constants in some hardy inequalities," Journal of Mathematical Inequalities, vol. 9, no. 2, pp. 437-447, 2007.

[13] A. Hussain and N. Sarfraz, "Optimal weak type estimates for p-Adic Hardy operators," Analysis and Applications, vol. 12, no. 1, pp. 29-38, 2020.

[14] Y. Mizuta, A. Nekvinda, and T. Shimomura, "Optimal estimates for the fractional Hardy operator," Studia Mathematica, vol. 227, no. 1, pp. 1-19, 2015.

[15] T. L. Yee and K. P. Ho, "Hardy's inequalities and integral operators on Herz-Morrey spaces," Open Mathematics, vol. 18, no. 1, pp. 106-121, 2020.

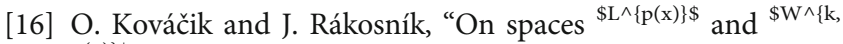
$\mathrm{p}(\mathrm{x})\}$," Czechoslovak mathematical journal, vol. 41, no. 4, pp. 592-618, 1991.

[17] D. Cruz-Uribe, A. Fiorenza, J. M. Martell, and C. Perez, "The boundedness of classical operators on variable $\mathrm{L}^{\mathrm{p}}$ spaces," Annales-Academiae Scientiarum Fennicae Mathematica, vol. 31, no. 1, pp. 239-264, 2006.

[18] D. Cruz-Uribe, L. Diening, and A. Fiorenza, "A new proof of the boundedness of maximal operators on variable Lebesgue spaces," Bollettino della Unione Matematica Italiana, vol. 2, no. 1, pp. 151-173, 2009.

[19] L. Diening, L. Harjulehto, P. Hasto, and P. Ruicka, "Lebesgue and Sobolev spaces with variable exponents," vol. 2011, Springer, Heidelberg, 2017.

[20] D. Cruz-Uribe and D. V. Fiorenza, "Variable Lebesgue spaces. Foundations and harmonic analysis," Springer Science \& Business Media, Heidelberg, 2013.

[21] D. Cru-Uribe, A. Fiorenza, and C. Neugebauer, "The maximal function on variable $\mathrm{L}^{\mathrm{p}}$ spaces," Annales-Academiae Scientiarum Fennicae Mathematica, vol. 28, pp. 223-238, 2003.

[22] B. Muckenhoupt, "Weighted norm inequalities for the Hardy maximal function," Transactions of the American Mathematical Society, vol. 165, pp. 207-226, 1972.

[23] L. Diening and P. Hästö, Muckenhoupt weights in variable exponent spaces, 2008, http://www.problemsolving.fi/pp/ p75submit.pdf.
[24] D. Cruz-Uribe, L. Diening, and P. Hästö, “The maximal operator on weighted variable Lebesgue spaces," Fractional Calculus and Applied Analysis, vol. 14, no. 3, pp. 361-374, 2011.

[25] D. Cruz-Uribe, A. Fiorenza, and C. J. Neugebauer, "Weighted norm inequalities for the maximal operator on variable Lebesgue spaces," Journal of Mathematical Analysis and Applications, vol. 394, no. 2, pp. 744-760, 2012.

[26] D. Cruz-Uribe and L. A. Wang, "Extrapolation and weighted norm inequalities in the variable Lebesgue spaces," Transactions of the American Mathematical Society, vol. 369, no. 2, pp. 1205-1235, 2017.

[27] M. Izuki, E. Nakai, and Y. Sawano, "Wavelet characterization and modular inequalities for weighted Lebesgue spaces with variable exponent," Annales-Academiae Scientiarum Fennicae Mathematica, vol. 40, pp. 551-571, 2015.

[28] M. Izuki, "Remarks on Muckenhoupt weights with variable exponent," Journal of Analysis and Applications, vol. 2, no. 1, pp. 27-41, 2013.

[29] M. Izuki, "Boundedness of sublinear operators on Herz spaces with variable exponent and application to wavelet characterization," Analysis Mathematica, vol. 36, no. 1, pp. 33-50, 2010.

[30] A. Almeida and D. Drihem, "Maximal, potential and singular type operators on Herz spaces with variable exponents," Journal of Mathematical Analysis and Applications, vol. 394, no. 2, pp. 781-795, 2012.

[31] S. Samko, "Variable exponent Herz spaces," Mediterranean Journal of Mathematics, vol. 10, no. 4, pp. 2007-2025, 2013.

[32] J. L. Wu and W. J. Zhao, "Boundedness for fractional Hardytype operator on variable-exponent Herz Morrey spaces," Kyoto Journal of Mathematics, vol. 56, no. 4, pp. 831-845, 2016.

[33] A. Hussain and G. Gao, "Status of day care laparoscopic appendectomy in developing countries," International Scholarly Research Notices, vol. 2014, 5 pages, 2014.

[34] A. Meskhi, H. Rafeiro, and M. A. Zaighum, "Central Calderón-Zygmund operators on Herz-type Hardy spaces of variable smoothness and integrability," Annals of Functional Analysis, vol. 9, no. 3, pp. 310-321, 2018.

[35] K. P. Ho, "Extrapolation to Herz spaces with variable exponents and applications," Revista Matemática Complutense, vol. 33, no. 2, pp. 437-463, 2020.

[36] F. Gürbüz, "Some estimates for generalized commutators of rough fractional maximal and integral" operators on generalized weighted Morrey spaces," Canadian Mathematical Bulletin, vol. 60, no. 1, pp. 131-145, 2017.

[37] F. Gürbüz, S. Ding, H. Han, and P. Long, "Characterizations of rough fractional-type integral Op-" erators on variable exponent vanishing Morrey-type spaces," in Topics in Contemporary Mathematical Analysis and Applications, H. Dutta, Ed., pp. 95-123, CRC Press, 2021.

[38] F. Gürbüz, S. Ding, H. Han, and P. Long, "Norm inequalities on variable exponent vanishing Morrey" type spaces for the rough singular type integral operators," International Journal of Nonlinear Sciences and Numerical Simulation, vol. 2020, pp. 1-19, 2020.

[39] M. Izuki and T. Noi, "Boundedness of fractional integrals on weighted Herz spaces with variable exponent," Journal of Inequalities and Applications, vol. 2016, 15 pages, 2016.

[40] M. Izuki and T. Noi, "An intrinsic square function on weighted Herz spaces with variable exponent," Journal of Mathematical Inequalities, vol. 11, pp. 799-816, 2017. 
[41] M. Izuki and T. Noi, "Two weighted Herz Spaces with variable exponents," Bulletin of the Malaysian Mathematical Sciences Society, vol. 43, no. 1, pp. 169-200, 2020.

[42] M. Izuki, "Boundedness of vector-valued sublinear operators on Herz-Morrey spaces with variable exponent," Mathematical Sciences Research Journal, vol. 13, pp. 243-253, 2009.

[43] L. Wang and L. Shu, "Boundedness of some sublinear operators on weighted variable Herz-Morrey spaces," Journal of Mathematical Inequalities, vol. 12, pp. 31-42, 2007.

[44] S. R. Wang and J. S. Xu, "Weighted norm inequality for bilinear Calderon-Zygmund operators on HerzMorrey spaces with variable exponents," Journal of Inequalities and Applications, vol. 2019, no. 1, p. 23, 2019.

[45] C. S. Herz, "Lipschitz spaces and Bernstein's theorem on absolutely convergent Fourier transforms," Journal of Mathematics and Mechanics, vol. 18, pp. 283-323, 1968.

[46] S. Lu and L. Xu, "Boundedness of rough singular integral operatorson the homogeneous Morrey-Herz spaces," Hokkaido Mathematical Journal, vol. 34, no. 2, pp. 299-314, 2005.

[47] C. Bennett and R. C. Sharpley, Interpolation of Operators, Academic Press, Boston, 1988.

[48] A. Y. Karlovich and I. M. Spitkovsky, "The Cauchy singular integral operator on weighted variable Lebesgue spaces," in Concrete Operators, Spectral Theory, Operators in Harmonic Analysis and Approximation, vol. 236, pp. 275-291, Springer, Basel, 2014.

[49] L. Grafakos, Modern Fourier analysis, Springer, 2nd edition, 2008.

[50] Y. Sawano, G. Di Fazio, and D. I. Hakim, "Introduction and applications to integral operators and PDE's," in Monographs and Research Notes in Mathematics, CRC Press, Boca Raton, FL, 2020. 